\title{
Hommage à Christian Petiau
}

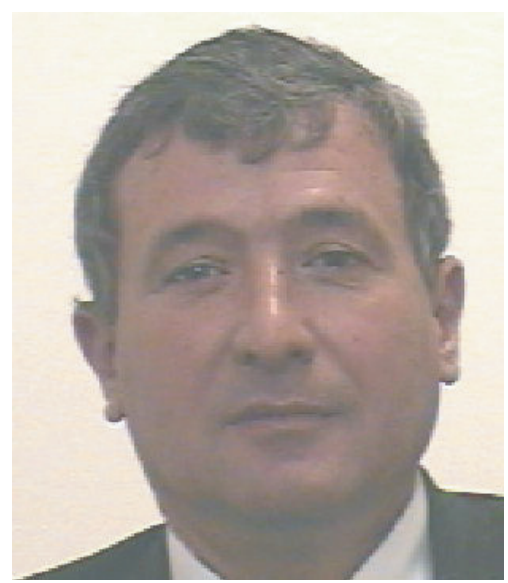

Christian PETIAU est né le 26 décembre 1942 à Paris.

Il sort diplômé de Sup'Aéro en 1967, puis effectue son service militaire à la DTE (Direction Technique des Engins), jusqu'en 1969, où il rentre chez Dassault Aviation (à l'époque GAMD). Il entame sa carrière pour travailler sur le mercure au bureau d'études, jusqu'en 1970. Il rentre alors au bureau de calcul. Il y crée le logiciel ELFINI de Dassault Aviation qui a regroupé progressivement tous les outils de l'analyse structurale des avions:

- méthode d'éléments finis linéaires et non-linéaires;

- critères de résistance en statique et en fatigue, propagation de fissures;

- calculs dynamiques, vibrations, chocs, impacts, ..., couplage avion-atterrisseurs, etc. ;

- aéroélasticité stationnaire \& instationnaire (Flutter);

- vibro-acoustique, aéro-acoustique;

- thermique, thermoélasticité;

- intégration avec l'outil de « CAO » (CATIA) ;

- optimisation structurale mathématique (intégrant des « contraintes » venant de tous les types d'analyse précédents);

- identification mathématique des modèles numériques précédents sur résultats d'essais.

Une partie de cet outil sera commercialisé dans CATIA par Dassault Systèmes (notamment chez Boeing), et directement par Dassault Aviation chez les Indiens pour réaliser le LCA (Light Combat Aircraft).

Il participe à la conception de tous les avions de la société depuis : Mercure, Falcon(s), Mirage 2000/4000, Rafale A, Rafale C, et navette Hermès, et Falcon F7X.

Il conçoit le système de « Protections Thermiques et Thermostructures » (TPS) de la Navette Européenne « Hermès », pour lequel il obtiendra un brevet (Brevet des principes originaux des Tuiles Hermès, notamment de leur principe de fixation par boulonnage).

Il devient chef de service en 1976, puis chef de département en 1984, et chef de la division structure au centre spatial dassault à Toulouse.

En 1992, il rentre à Saint-Cloud pour devenir expert émérite à la direction prospective et recherche, conseiller scientifique pour les affaires de structure et chargé de missions « transversales » diverses, notamment mise en place et réalisation :

- plan «modélisation et contrôle de l'avion souple»;

- plan « acoustique » de réduction des bruits internes et externes des avions Falcon;

- plan « MDO $»$ (multidisciplinary design optimisation).

En 2002, il devient directeur adjoint, avec les mêmes fonctions, de cette direction.

Il laisse de nombreuses publications aussi bien sur le calcul des structures linéaire et non-linéaire, la méthode d'éléments finis, l'aéroélasticité, le calcul des charges, l'identification de modèle, l'aéroacoustique, l'optimisation, les thermostructures.

Il a de plus occupé différentes positions :

- président du club CRIN « bureau d'étude du futur » (de 1993 à1997);

- président du conseil scientifique de l'Association Française de Mécanique (1999-2003);

- représentant Dassault au comité technique du GIFAS (depuis 2003) ;

- membre émérite de l'AAAF, après avoir participé aux comités techniques durant de longues années, et membre de l'AIAA.

Christian Petiau s'est énormément impliqué dans les relations sciences industries dans le domaine des matériaux et des structures. Craint et respecté pour ses positions claires, tranchées et pertinentes vis-à-vis des travaux académiques, Christian Petiau a joué un rôle moteur dans l'essor de la communauté du calcul des structures à travers son implication forte et sans faille tout d'abord dans le GRECO-GIS « calcul des structures et intelligence artificelle » puis dans l'association CSMA, émanation de ce GRECO-GIS. Christian Petiau a également joué un rôle majeur dans l'animation scientifique de l'Association Française de Mécanique et est à l'origine d'un des projets fédérateurs issus de l'AFM et consacré à l'optimisation pluri-disciplinaire.

Sa notoriété était établie au niveau national et mondial.

Christian PETIAU nous a quittés dans le 17 mars 2006, à la suite d'une longue maladie. 